\title{
INCOMPLETE PREFERENCES UNDER UNCERTAINTY: INDECISIVENESS IN BELIEFS VERSUS TASTES
}

\author{
By Efe A. OK, Pietro OrToleva, AND Gil Riella ${ }^{1}$
}

\begin{abstract}
We investigate the classical Anscombe-Aumann model of decision-making under uncertainty without the completeness axiom. We distinguish between the dual traits of "indecisiveness in beliefs" and "indecisiveness in tastes." The former is captured by the Knightian uncertainty model, the latter by the single-prior expected multi-utility model. We characterize axiomatically the latter model. Then we show that, under independence and continuity, these two models can be jointly characterized by means of a partial completeness property.
\end{abstract}

KEYWORDS: Incomplete preferences, the Anscombe-Aumann model, Knightian uncertainty.

\section{INTRODUCTION}

IN THE CONTEXT of uncertainty, there appear to be two main sources for the incompleteness of the preferences of a decision maker. First, a person may find it impossible to compare the desirability of two acts due to the variation of the promises of these acts across states, because, for some reason, she cannot formulate a "precise guess" about the likelihood of the states of the world. This sort of an incompleteness of preferences, which may be called indecisiveness in beliefs, is what Bewley's well known model of Knightian uncertainty is primed to capture. Second, even if one is able to assess, subjectively, the likelihood of each state, her preferences over acts might still be incomplete, due to a possible inability to compare some certain outcomes. In turn, such sort of an incompleteness of preferences, which may be called indecisiveness in tastes, was what Aumann (1962) has aimed to capture. Put succinctly, the main objective of the present paper is to understand how we may be able to distinguish between one's indecisiveness in her beliefs and in her tastes from a behavioral perspective.

We adopt the framework of the classical Anscombe-Aumann (1963) model with a finite state space $\Omega$ and a compact (metric) prize space $X$. Our interest in this paper is confined to those preference relations (on the space of so-called horse race lotteries) that satisfy the Independence, Continuity, and Monotonicity Axioms. A classical result in this setup is the Anscombe-Aumann expected utility theorem which shows that any such preference relation admits a single-prior expected single-utility representation, provided that this relation is complete. In turn, Bewley (2002) showed that if we require in this theorem only

\footnotetext{
${ }^{1}$ Various discussions with Ozgur Evren and Peter Wakker have contributed to this work; we gratefully acknowledge our intellectual debt to them. We also thank the co-editor and three referees for their substantial comments, expository and otherwise, concerning this paper. Finally, we acknowledge the financial support of the C. V. Starr Center for Applied Economics at New York University.
} 
that the preference relation be complete on the set of all constant acts, then that relation admits a multi-prior expected single-utility representation. The first result of the present paper is that, if we instead replace the completeness assumption with the postulate that subjects can reduce subjective uncertainty to objective uncertainty, at least locally, then we obtain a single-prior expected multi-utility representation; this may be viewed as the "dual" of the Knightian uncertainty model.

We prove this theorem, indirectly, by first investigating the preferences that exhibit either one or the other type of indecisiveness. This is in line with some earlier work-see García del Amo and Ríos Insua (2002), Seidenfeld, Schervish, and Kadane (1995), and Nau (2006) — that focused on preferences that exhibit indecisiveness in beliefs and tastes simultaneously. We show here that a necessary condition for both forms of incompleteness to hold at the same time is that preferences violate a basic comparability property, which we call the Weak Reduction Axiom. Put precisely, a preference relation that satisfies the Independence, Continuity, and Weak Reduction Axioms can exhibit only indecisiveness in beliefs or indecisiveness in tastes (but not both). As an immediate consequence of this result, we obtain the aforementioned characterization of the dual Knightian uncertainty model. Furthermore, we show that this approach allows us to obtain the Knightian uncertainty model itself as a consequence of a certain type of reduction of subjective uncertainty to objective uncertainty, thereby providing a unified behavioral basis for both of these models.

\section{PRELIMINARIES}

We work within the classical Anscombe-Aumann setup with a finite state space. In what follows, $\Omega$ stands for a finite (nonempty) set of states of the world, $X$ stands for a compact metric prize space, and $\Delta(X)$ stands for the set of all Borel probability measures (lotteries) on $X$. As usual, we metrize $\Delta(X)$ in such a way that metric convergence on it coincides with weak convergence of Borel probability measures.

The linear space of all continuous real maps on $X$ is denoted as $\mathbf{C}(X)$; we view this space as normed by the sup-norm throughout the exposition. The expectation of any map $u$ in $\mathbf{C}(X)$ with respect to a probability measure $p$ in $\Delta(X)$ is denoted by $\mathbf{E}(u, p)$, that is,

$$
\mathbf{E}(u, p):=\int_{X} u d p .
$$

An act (or horse race lottery) in this setup is a function that maps the state space $\Omega$ into the space $\Delta(X)$ of lotteries. The set of all acts, denoted by $\mathcal{F}$, is, therefore, $\Delta(X)^{\Omega}$. In what follows, for any nonempty subset $S$ of $\Omega$, we denote by $\mathbf{1}_{S}$ the indicator function of $S$ on $\Omega$. Thus, for any lottery $p \in \Delta(X)$ and act 
$f \in \mathcal{F}$, the act that yields $p$ if any one of the states in $S$ occurs, and $f(\omega)$ at any other state $\omega$, is written as $p \mathbf{1}_{S}+f \mathbf{1}_{\Omega \backslash S}$. In particular, the constant act that yields the lottery $p$ at every state of the world can be written as $p \mathbf{1}_{\Omega}$.

A preference relation $\succsim$ in the present framework is a preorder (i.e., a reflexive and transitive binary relation) on $\mathcal{F}$. (As usual, the asymmetric part of this preorder is denoted by $\succ$, and its symmetric part by $\sim$.) The following are three standard postulates imposed on $\succsim$.

THE INDEPENDENCE AXIOM: For any acts $f, g$, and $h$ in $\mathcal{F}$, and $0<\lambda \leq 1$,

$$
f \succsim g \text { implies } \lambda f+(1-\lambda) h \succsim \lambda g+(1-\lambda) h .
$$

THE CONTINUITY AXIOM: $\succsim$ is a closed subset of $\mathcal{F} \times \mathcal{F} .{ }^{2}$

The Monotonicity Axiom: For any acts $f$ and $g$ in $\mathcal{F}$, if $f(\omega) \mathbf{1}_{\Omega} \succsim g(\omega) \mathbf{1}_{\Omega}$ for every state $\omega$ in $\Omega$, then $f \succsim g$.

The Anscombe-Aumann expected utility theorem says that a complete preference relation $\succsim$ on $\mathcal{F}$ that satisfies the Independence, Continuity, and Monotonicity Axioms can be represented by an aggregate utility function of the form

$$
f \mapsto \sum_{\omega \in \Omega} \mu(\omega) \mathbf{E}(u, f(\omega)),
$$

where $\mu$ is a probability distribution on $\Omega$-interpreted as one's prior beliefs about the states of the world-and $u$ is a continuous real function on $X-$ interpreted as one's von Neumann-Morgenstern utility function over (riskless) prizes.

\section{NOTIONS OF INCOMPLETENESS}

\subsection{Indecisiveness in Beliefs}

The most well known weakening of completeness in the Anscombe-Aumann framework is the requirement that preferences be complete at least over constant acts:

THE C-COMPLETENESS AXIOM: For any lotteries $p$ and $q$ in $\Delta(X)$,

$$
\text { either } \quad p \mathbf{1}_{\Omega} \succsim q \mathbf{1}_{\Omega} \text { or } \quad q \mathbf{1}_{\Omega} \succsim p \mathbf{1}_{\Omega} .
$$

\footnotetext{
${ }^{2}$ That is, for any convergent sequences $\left(f_{m}\right)$ and $\left(g_{m}\right)$ in $\mathcal{F}$, with $f_{m} \succsim g_{m}$ for each $m$, we have $\lim f_{m} \succsim \lim g_{m}$. We note that, when $X$ is a finite set, all theorems in this paper remain valid if this continuity property is replaced by the much weaker requirement that the sets $\{\alpha: \alpha f+(1-\alpha) g \succsim$ $h\}$ and $\{\alpha: h \succsim \alpha f+(1-\alpha) g\}$ are closed in $[0,1]$ for any $f, g$, and $h$ in $\mathcal{F}$.
} 
When combined with the Independence, Continuity, and Monotonicity, Axioms, the C-Completeness Axiom initiates the following notion of expected multi-utility representation:

DEFINITION: A preference relation $\succsim$ on $\mathcal{F}$ is said to admit a multi-prior expected single-utility representation if there exists a nonempty subset $\mathcal{M}$ of $\Delta(\Omega)$ and a function $u \in \mathbf{C}(X)$ such that, for any acts $f$ and $g$ in $\mathcal{F}$, we have $f \succsim g$ if and only if (iff)

$$
\sum_{\omega \in \Omega} \mu(\omega) \mathbf{E}(u, f(\omega)) \geq \sum_{\omega \in \Omega} \mu(\omega) \mathbf{E}(u, g(\omega)) \quad \text { for every } \mu \in \mathcal{M} .
$$

Using this terminology, we can rephrase Bewley's (2002) characterization as follows:

BEWLEY'S EXPECTED UTILITY THEOREM: A preference relation $\succsim$ on $\mathcal{F}$ satisfies the Independence, Continuity, Monotonicity, and C-Completeness Axioms if, and only if, it admits a multi-prior expected single-utility representation.

Bewley's model is a model of indecisiveness in beliefs. In this model, due to C-completeness, the agent's tastes over the lotteries in $\Delta(X)$ are presumed complete (and represented by a single von Neumann-Morgenstern utility function). Instead, the indecisiveness of this individual pertains to her beliefs, and manifests itself in the multiplicity of the priors in the representation (1). ${ }^{3}$

\subsection{Indecisiveness in Tastes}

Despite-or perhaps, because of-the influence of the Knightian uncertainty model in decision theory and its applications, the literature does not provide a "dual" to this model, one that would concentrate on the incompleteness of the tastes of a decision maker, instead of on that of her beliefs. While, with its origins going back to the seminal contribution of Aumann (1962), there is now a fairly mature literature on modeling the incompleteness of tastes of an individual over certain or risky prospects, such models are seldom considered in the context of uncertainty. Indeed, a model that focuses only on this type of indecisiveness would have the following form of expected utility representation:

DEFINITION: A preference relation $\succsim$ on $\mathcal{F}$ is said to admit a single-prior expected multi-utility representation if there exist a probability distribution

\footnotetext{
${ }^{3}$ Bewley's model has found many applications in economics. For instance, Rigotti and Shannon (2005) and Kelsey and Yalcin (2007) used this model in the context of finance, Lopomo, Rigotti, and Shannon (2009) in the context of mechanism design, and Ghirardato and Katz (2006) in the context of voting theory.
} 
$\mu \in \Delta(\Omega)$ and a nonempty subset $\mathcal{U}$ of $\mathbf{C}(X)$ such that, for any acts $f$ and $g$ in $\mathcal{F}$, we have $f \succsim g$ iff

$$
\sum_{\omega \in \Omega} \mu(\omega) \mathbf{E}(u, f(\omega)) \geq \sum_{\omega \in \Omega} \mu(\omega) \mathbf{E}(u, g(\omega)) \quad \text { for every } u \in \mathcal{U} .
$$

In this case, we say that $(\mu, \mathcal{U})$ is a single-prior expected multi-utility representation for $\succsim$.

This utility representation notion extends the so-called expected multi-utility representation of preferences over lotteries - see Dubra, Maccheroni, and Ok (2004) - to the context of preferences over uncertain acts in a straightforward manner. It models an agent who is confident in assessing the likelihood of the states of nature, but who is nevertheless unable to compare some lotteries with assurance.

An axiomatization of this model requires finding a behavioral postulate that would capture a dual form of C-completeness; such a postulate should allow for incompleteness of tastes, but not of beliefs. To formulate the property we will use for this purpose, we need the following notation.

NOTATION: For any act $f$ in $\mathcal{F}$ and any probability distribution $\alpha$ in $\Delta(\Omega)$, we write $f^{\alpha}$ to denote the constant act that yields the lottery $\sum_{\omega \in \Omega} \alpha(\omega) f(\omega)$ at every state, that is,

$$
f^{\alpha}:=\left(\sum_{\omega \in \Omega} \alpha(\omega) f(\omega)\right) \mathbf{1}_{\Omega} .
$$

Now we can introduce the following axiom.

The Reduction Axiom: For any act $f \in \mathcal{F}$, there is an $\alpha_{f} \in \Delta(\Omega)$ such that $f^{\alpha_{f}} \sim f$.

This axiom posits that the agent can always reduce subjective uncertainty to objective uncertainty by suitably mixing the outcomes of a given act. From this point of view, we can view the Reduction Axiom as the "local" formulation of the notion of probabilistic sophistication introduced by Machina and Schmeidler (1992). While probabilistic sophistication of a preference relation $\succsim$ on $\mathcal{F}$ demands that there is an $\alpha \in \Delta(\Omega)$ such that $f^{\alpha} \sim f$ for every act $f$ in $\mathcal{F}$, the Reduction Axiom allows for the nature of the mixing $\alpha$ to vary across the acts in $\mathcal{F}$.

We may now characterize the aforementioned "dual" of the Knightian uncertainty model:

THEOREM 1: A preference relation $\succsim$ on $\mathcal{F}$ satisfies the Independence, Continuity, and Reduction Axioms if, and only if, $\succsim$ admits a single-prior expected multi-utility representation. 
REMARK-Uniqueness of Single-Prior Expected Multi-Utility Representation: There is a sense in which a single-prior expected multi-utility $(\mu, \mathcal{U})$ for a preference relation on $\mathcal{F}$ is unique. This is characterized in Appendix A.2.

\section{CONNECTING THE TWO MODELS OF INCOMPLETE PREFERENCES}

\subsection{A Unification Theorem}

The notions of multi-prior expected single-utility representation and singleprior expected multi-utility representation correspond to two complementary sources of incompleteness in the context of the Anscombe-Aumann framework. This is formalized by the two axioms that help characterize these models, namely, the axioms of C-completeness and reduction. However, ostensibly, these two properties posit rather different sorts of behavioral restrictions on a preference relation. In particular, it is not clear in which sense one can think of these properties as special cases of a more basic notion of "partial completeness."

This question amounts to identifying the nature of "completeness" shared by both the Knightian uncertainty model and its dual. One possible answer to it stems from the fact that, in the context of either of these models, for every act $f$ there is at least one way of reducing subjective uncertainty to risk (by mixing the outcomes associated with $f$ ) so that the resulting constant act is comparable, in fact, preferred, to $f$. This suggests the following axiom.

THE WEAK REDUCTION AXIOM: For any act $f \in \mathcal{F}$, there is an $\alpha_{f} \in \Delta(\Omega)$ such that $f^{\alpha_{f}} \succsim f$.

The Weak Reduction Axiom is clearly a weakening of the Reduction Axiom. At the same time, it is also implied by C-completeness and monotonicity. (C-completeness implies that, for any act $f$, there is an $\omega^{*} \in \Omega$ such that $f\left(\omega^{*}\right) \mathbf{1}_{\Omega} \succsim f(\omega) \mathbf{1}_{\Omega}$ for all $\omega \in \Omega$, and hence, by monotonicity, $f^{\alpha} \succsim f$, where $\alpha \in \Delta(\Omega)$ satisfies $\alpha\left(\omega^{*}\right)=1$.) Consequently, the Weak Reduction Axiom is satisfied both by the single-prior expected multi-utility model and by the multiprior expected single-utility model. The theorem below shows that, among the preference relations that satisfy independence and continuity, these two models are actually the only ones compatible with this property.

THEOREM 2: A preference relation $\succsim$ on $\mathcal{F}$ satisfies the Independence, Continuity, and Weak Reduction Axioms if, and only if, $\succsim$ admits either

(i) a multi-prior expected single-utility representation or

(ii) a single-prior expected multi-utility representation.

This result shows that weak reduction, together with independence and continuity, implies that only one of the two "extreme" models above could represent the preferences. Put differently, for both types of indecisiveness to take 
place at the same time, there must be some act $f$ such that there is no $\alpha$ in $\Delta(\Omega)$ with $f^{\alpha} \succsim f$. This means that the two forms of indecisiveness can coexist only at the expense of the agent's ability to reduce subjective uncertainty to an objective one and still be able to make comparisons.

Remark - Weak Reduction as a Partial Completeness Axiom: Despite its initial appearance, the power of the Weak Reduction Axiom does not stem from its allowance for reducing subjective uncertainty to risk in a "favorable" manner. Indeed, in Theorem 2, this property can be replaced by the alternative requirement that, for each $f$, there is an $\alpha_{f}$ in $\Delta(\Omega)$ such that $f \succsim f^{\alpha_{f}}$; or that, for each act $f$, there are $\bar{\alpha}_{f}$ and $\underline{\alpha}_{f}$ in $\Delta(\Omega)$ such that $f^{\bar{\alpha}_{f}} \succsim f \succsim f^{\underline{\alpha}}{ }$. (After all, Theorem 2 implies that either of these properties is equivalent to the Weak Reduction Axiom in the presence of independence and continuity.) It thus seems more appropriate to view this axiom as a postulate that restricts the extent of incompleteness of $\succsim$, and not one that imposes an intrinsic preference for risk over uncertainty. ${ }^{4}$

\subsection{Bewley's Expected Utility Theorem, Reconsidered}

Theorem 2 provides a new route toward deriving the two models of incomplete preferences we considered above. In particular, Bewley's theorem is an immediate consequence of this result. To see this, we first note that Theorem 2 entails an alternative characterization of the multi-prior expected single-utility model in which monotonicity is replaced by the Weak Reduction Axiom:

Proposition 1: A preference relation $\succsim$ on $\mathcal{F}$ satisfies the Independence, Continuity, Weak Reduction, and C-Completeness Axioms if, and only if, it admits a multi-prior expected single-utility representation.

It is not difficult to show that a preference relation on $\mathcal{F}$ that admits a singleprior expected multi-utility representation satisfies C-completeness iff it is complete. Thus, Proposition 1 is an immediate consequence of Theorem 2 and the Anscombe-Aumann theorem. In turn, as noted earlier, C-completeness and monotonicity jointly imply the Weak Reduction Axiom, so Bewley's theorem obtains as an immediate corollary of Proposition 1.

\subsection{Proving Theorem 1}

Theorem 1 is also an immediate consequence of Theorem 2. Indeed, it is plain that a preference relation $\succsim$ on $\mathcal{F}$ satisfies reduction and C-completeness

\footnotetext{
${ }^{4}$ In this respect, it seems more reasonable to consider a weaker completeness requirement: For any act $f \in \mathcal{F}$, there is an $\alpha_{f} \in \Delta(\Omega)$ such that $f^{\alpha_{f}}$ and $f$ are deemed comparable by $\succsim$. At present, we do not know if this property is equivalent to the Weak Reduction Axiom in the presence of independence and continuity.
} 
iff it is complete. Therefore, by Theorem $2, \succsim$ satisfies reduction, independence, and continuity iff either it is complete or it admits a single-prior expected multi-utility representation. By the Anscombe-Aumann theorem, the former case is a special case of the latter, so Theorem 1 is proved.

\section{4. "Reduction"-Style Axioms and Other Properties}

As the Reduction Axiom can be thought of as a particular completeness property, the structure of the axiomatizations given in Bewley's theorem and in Theorem 1 can be viewed as independence, continuity, and monotonicity plus a completeness property. Dually, we can think of the structure of these axiom systems as independence and continuity plus a reduction property. In other words, just like reduction is a completeness property, there is a sense in which $\mathrm{C}$-completeness is a reduction property (that tells us how certain nonconstant acts relate to certain constant acts). To see this, consider the following axiom.

The Weak Sure-Reduction Axiom: For any act $f \in \mathcal{F}$, there is an $\omega \in \Omega$ such that $f(\omega) \mathbf{1}_{\Omega} \succsim f$.

For any preference relation $\succsim$ on $\mathcal{F}$, we say that a state in $\Omega$ is $\succsim$-sure if, for every two acts $f$ and $g$ in $\mathcal{F}$, we have $f \sim g$ whenever $f(\omega)=g(\omega)$. We next show that in the nontrivial case where there are no $\succsim$-sure states in $\Omega$, and in the presence of the other axioms of Bewley's theorem, C-completeness coincides with the Weak Sure-Reduction Axiom.

PROPOSITION 2: Let $\succsim$ be a preference relation on $\mathcal{F}$ that satisfies the independence and continuity axioms, and assume that no state in $\Omega$ is $\succsim$-sure. Then, $\succsim$ satisfies $C$-completeness and monotonicity if, and only if, it satisfies the Weak Sure-Reduction Axiom. ${ }^{5}$

It is possible to deduce this fact as another consequence of Theorem 2. We have already seen in Section 4.1 that C-completeness and monotonicity imply the Weak Sure-Reduction Axiom. Conversely, let $\succsim$ be a preference relation that satisfies the independence and continuity as well as the Weak SureReduction Axiom. Then, by Theorem 2, $\succsim$ admits either a multi-prior expected single-utility or a single-prior expected multi-utility representation. In the former case, we are done, so assume the latter case, and let $(\mu, \mathcal{U})$ be a singleprior expected multi-utility for $\succsim$. Notice that monotonicity must hold. Now take any two lotteries $p$ and $q$ in $\Delta(X)$, fix a state $\omega \in \Omega$ with $\mu(\omega)>0$, and

\footnotetext{
${ }^{5}$ As it was the case with the Weak Reduction Axiom, this property should be viewed as one that allows reducing subjective uncertainty to risk in a "comparable" manner, not necessarily in a "favorable" manner. For instance, in Proposition 2, this property can be replaced by the alternative requirement that, for each $f$, there is an $\omega$ in $\Omega$ such that $f \succsim f(\omega) \mathbf{1}_{\Omega}$.
} 
consider the act $f:=p \mathbf{1}_{\{\omega\}}+q \mathbf{1}_{\Omega \backslash\{\omega\}}$. By weak sure-reduction, we have either $p \mathbf{1}_{\Omega} \succsim f$ or $q \mathbf{1}_{\Omega} \succsim f$. But $\mu(\omega)<1$, because $\omega$ is not $\succsim$-sure. Then, using the representation of $\succsim$ via $(\mu, \mathcal{U})$, one may readily check that the former statement holds iff $p \mathbf{1}_{\Omega} \succsim q \mathbf{1}_{\Omega}$, and the latter holds iff $q \mathbf{1}_{\Omega} \succsim p \mathbf{1}_{\Omega}$. Thus, $\succsim$ satisfies C-completeness, as we sought.

The models of multi-prior expected single-utility and single-prior expected multi-utility correspond to dual notions of representation. Combining Proposition 2 with Bewley's theorem, and comparing with Theorem 1, provides a "dual" outlook for the respective axiomatizations of these models as well. Adding weak sure-reduction to independence and continuity yields the former model (indecisiveness in beliefs), while adding reduction to them instead yields the latter (indecisiveness in tastes). These observations are summarized in Figure 1, which contains a Venn diagram that depicts the logical relation between the different axioms and representations, under the assumptions of independence and continuity.

In passing, we should mention that Nau (2006) and Galaabaatar and Karni (2012) have axiomatized, under the restriction of a finite prize space, representations that allow for indecisiveness in taste and beliefs simultaneously. The main axiom in Nau (2006) is a stronger version of the standard separability axiom, while the main property in Galaabaatar and Karni (2012) is, under independence and continuity, a strengthening of monotonicity. It can be shown that, under independence and continuity, weak reduction is stronger than both of these properties. This means that, in some sense, weak reduction not only

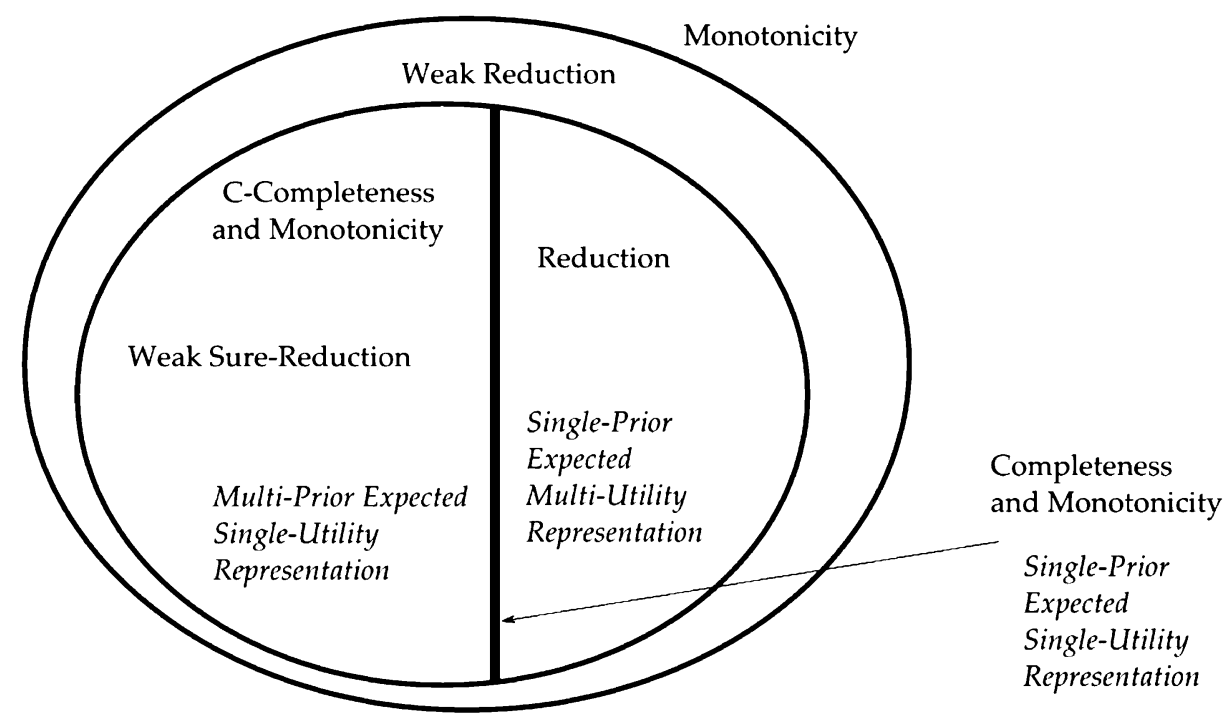

FIGURE 1.-Relation between axioms and representations assuming independence, continuity, and no $\succsim$-sure state. 
implies the strengthenings of separability and monotonicity above, but it also contains an additional form of partial completeness. ${ }^{6}$

\section{A SKETCH OF THE PROOF FOR THEOREM 2}

This section is devoted to proving Theorem 2. The "if" part of this result is straightforward, so we concentrate here on its "only if" part alone. To this end, let $\succsim$ be a preference relation on $\mathcal{F}$ that satisfies the Independence, Continuity, and Weak Reduction Axioms.

We divide the main argument into three steps. The first step is a bit technical and is treated in full only in Appendix A.1. The arguments in the subsequent two steps are meant to be complete.

Step 1. There exists a nonempty subset $\mathcal{U}$ of $\mathbf{C}(X \times \Omega)$ such that, for any acts $f$ and $g$ in $\mathcal{F}$, we have $f \succsim g$ iff

$$
\sum_{\omega \in \Omega} \mathbf{E}(U(\cdot, \omega), f(\omega)) \geq \sum_{\omega \in \Omega} \mathbf{E}(U(\cdot, \omega), g(\omega)) \quad \text { for every } U \in \mathcal{U} .
$$

Theorem 0 in the Appendix shows that this statement is equivalent to the Independence and Continuity Axioms alone. ${ }^{8}$

Step 2. There exists a nonempty subset $\mathcal{H}$ of $\Delta(\Omega) \times \mathbf{C}(X)$ such that, for any acts $f$ and $g$ in $\mathcal{F}$, we have $f \succsim g$ iff

$$
\sum_{\omega \in \Omega} \mu(\omega) \mathbf{E}(u, f(\omega)) \geq \sum_{\omega \in \Omega} \mu(\omega) \mathbf{E}(u, g(\omega)) \quad \text { for every }(\mu, u) \in \mathcal{H} .
$$

We refer to the representation in (3) as a multi-prior expected multi-utility representation. In words, Step 2 says that, whenever $\succsim$ satisfies the Weak Reduction Axiom, each of the state-dependent utility functions found in Step 1 can in fact be made state-independent. ${ }^{9}$ This is an easy consequence of the following observation:

\footnotetext{
${ }^{6}$ However, while the properties in Nau (2006) and Galaabaatar and Karni (2012) mentioned above cannot be seen as partial completeness properties, both papers are not free of assumptions of this kind. In particular, both impose the existence of a best and a worst option, which, especially when coupled with independence, induces quite a bit of comparability on the part of the agent.

${ }^{7}$ Our earlier proof of this result was rather clumsy. One of the referees of this journal has kindly suggested a simpler argument. It is this argument that we present in this section.

${ }^{8}$ In the context of a finite prize space, the same result was proved in Nau (2006). García del Amo and Ríos Insua (2002) presented a related result, when $X$ and $\Omega$ are compact subsets of a Euclidean space.

${ }^{9}$ When $\succsim$ is complete, independence and continuity imply that it is represented by a single state-dependent expected-utility function (cf. Kreps (1988)). In turn, the Anscombe-Aumann theorem shows that monotonicity is enough to obtain state-independence in this case. This is no longer true when $\succsim$ is not complete. That is, if we replaced weak reduction by monotonicity, we would not obtain the representation in (3). See Seidenfeld, Schervish, and Kadane (1995) and $\mathrm{Nau}$ (2006) for related counterexamples.
} 
Claim 1: For each $U$ in $\mathcal{U}$, there is a state $\omega_{U} \in \Omega$ such that every $U(\cdot, \omega)$ is a nonnegative affine transformation of $U\left(\cdot, \omega_{U}\right)$.

PROOF: If $U(\cdot, \omega)$ is constant for each $\omega \in \Omega$, then the claim is trivially true. Suppose, then, there is an $\omega_{1} \in \Omega$ such that $U\left(\cdot, \omega_{1}\right)$ is not constant. We wish to show that $U(\cdot, \omega)$ is a nonnegative affine transformation of $U\left(\cdot, \omega_{1}\right)$ for any $\omega \in \Omega \backslash\left\{\omega_{1}\right\}$. To derive a contradiction, suppose there is a state $\omega_{2} \in \Omega$, distinct from $\omega_{1}$, such that $U\left(\cdot, \omega_{2}\right)$ is not a nonnegative affine transformation of $U\left(\cdot, \omega_{1}\right)$. Then, these functions are the von Neumann-Morgenstern utilities for two different and nontrivial risk preferences over $\Delta(X)$. It follows that there exist two lotteries $p$ and $q$ such that

$$
\begin{aligned}
& \mathbf{E}\left(U\left(\cdot, \omega_{1}\right), p\right)>\mathbf{E}\left(U\left(\cdot, \omega_{1}\right), q\right) \text { and } \\
& \mathbf{E}\left(U\left(\cdot, \omega_{2}\right), p\right)<\mathbf{E}\left(U\left(\cdot, \omega_{2}\right), q\right) .{ }^{10}
\end{aligned}
$$

We now consider the act $f \in \mathcal{F}$ defined as

$$
f(\omega):= \begin{cases}p, & \text { if } \mathbf{E}(U(\cdot, \omega), p) \geq \mathbf{E}(U(\cdot, \omega), q), \\ q, & \text { otherwise. }\end{cases}
$$

It follows readily from the structure of $f$ and (4) that

$$
\sum_{\omega \in \Omega} \mathbf{E}(U(\cdot, \omega), f(\omega))>\sum_{\omega \in \Omega} \mathbf{E}\left(U(\cdot, \omega), f^{\alpha}(\omega)\right) \quad \text { for every } \alpha \in \Delta(\Omega) .
$$

Therefore, $f^{\alpha} \succsim f$ is false for every $\alpha \in \Delta(\Omega)$, contradicting the Weak Reduction Axiom.

Q.E.D.

Step 2 is now completed by means of a standard normalization argument. Take any $U$ in $\mathcal{U}$. By Claim 1 , there are two maps $\alpha_{U}: \Omega \rightarrow \mathbb{R}_{+}$and $\beta_{U}: \Omega \rightarrow \mathbb{R}$ such that $\alpha_{U}\left(\omega_{U}\right)=1, \beta_{U}\left(\omega_{U}\right)=0$, and

$$
U(\cdot, \omega)=\alpha_{U}(\omega) U\left(\cdot, \omega_{U}\right)+\beta_{U}(\omega) \quad \text { for each } \omega \in \Omega .
$$

Defining $\mu_{U} \in \Delta(\Omega)$ by $\mu_{U}(\omega):=\alpha_{U}(\omega) / \sum_{\tau \in \Omega} \alpha_{U}(\tau)$, and using Step 1, we find that $\mathcal{H}:=\left\{\left(\mu_{U}, U\left(\cdot, \omega_{U}\right)\right): U \in \mathcal{U}\right\}$ is a multi-prior expected multi-utility for $\succsim$.

\footnotetext{
${ }^{10}$ More precisely, the fact that $U\left(\cdot, \omega_{2}\right)$ is not a nonnegative affine transformation of $U\left(\cdot, \omega_{1}\right)$
} entails that there exist two lotteries $p^{\prime}$ and $q^{\prime}$ such that

$$
\mathbf{E}\left(U\left(\cdot, \omega_{1}\right), p^{\prime}\right) \geq \mathbf{E}\left(U\left(\cdot, \omega_{1}\right), q^{\prime}\right) \quad \text { and } \quad \mathbf{E}\left(U\left(\cdot, \omega_{2}\right), p^{\prime}\right)<\mathbf{E}\left(U\left(\cdot, \omega_{2}\right), q^{\prime}\right) .
$$

As $U\left(\cdot, \omega_{1}\right)$ is not constant, it is clear that either $\mathbf{E}\left(U\left(\cdot, \omega_{1}\right), P\right)>\mathbf{E}\left(U\left(\cdot, \omega_{1}\right), q^{\prime}\right)$ for some lottery $P$ on $X$ or $\mathrm{E}\left(U\left(\cdot, \omega_{1}\right), p^{\prime}\right)>\mathbf{E}\left(U\left(\cdot, \omega_{1}\right), Q\right)$ for some lottery $Q$ on $X$. Defining $p$ to be $(1-\lambda) p^{\prime}+$ $\lambda P$ for small enough $\lambda>0$ and setting $q=q^{\prime}$ in the first case, and defining $q$ to be $(1-\lambda) q^{\prime}+\lambda Q$ for small enough $\lambda>0$ and setting $p=p^{\prime}$ in the second, we obtain (4). 
Step 3. $\succsim$ admits either a multi-prior expected single-utility representation or a single-prior expected multi-utility representation.

To see this, assume that $\succsim$ admits neither a multi-prior expected single-utility nor a single-prior expected multi-utility representation. Then, there exist two pairs $(\mu, u)$ and $(\sigma, v)$ in the collection $\mathcal{H}$ we found in Step 2 such that $\mu \neq \sigma$, $u$ and $v$ are not constant, and $v$ is not a positive affine transformation of $u$. Then, there exist an event $A \subseteq \Omega$ such that $\mu(A)>\sigma(A)$, and lotteries $p, q \in$ $\Delta(X)$ such that $\mathbf{E}(u, p) \geq \mathbf{E}(u, q)$ and $\mathbf{E}(v, p)<\mathbf{E}(v, q)$. Furthermore, as in the proof of Claim 1 , it is without loss of generality to posit that $\mathbf{E}(u, p)>$ $\mathbf{E}(u, q)$ here.

The key observation is that, for the act $f:=p \mathbf{1}_{A}+q \mathbf{1}_{\Omega \backslash A}$ and any $\alpha \in \Delta(\Omega)$, we have

$$
\sum_{\omega \in \Omega} \mu(\omega) \mathbf{E}(u, f(\omega)) \leq \sum_{\omega \in \Omega} \mu(\omega) \mathbf{E}\left(u, f^{\alpha}(\omega)\right) \quad \text { iff } \quad \mu(A) \leq \alpha(A),
$$

whereas

$$
\sum_{\omega \in \Omega} \sigma(\omega) \mathbf{E}(v, f(\omega)) \leq \sum_{\omega \in \Omega} \sigma(\omega) \mathbf{E}\left(v, f^{\alpha}(\omega)\right) \quad \text { iff } \quad \sigma(A) \geq \alpha(A) .
$$

But, by the Weak Reduction Axiom, there is an $\alpha \in \Delta(\Omega)$ such that $f^{\alpha} \succsim f$. Thus, in view of the representation of $\succsim,(5)$ and (6) yield $\sigma(A) \geq \mu(A)$, a contradiction. Our proof of Theorem 2 is now complete.

\section{APPENDIX}

\section{A.1. On Step 1 of the Proof of Theorem 2}

The argument we gave in Step 1 of the proof of Theorem 2 above is based on the following additive representation theorem.

THEOREM 0: $A$ preference relation $\succsim$ on $\mathcal{F}$ satisfies the Independence and Continuity Axioms if, and only if, there exists a nonempty convex subset $\mathcal{U}$ of $\mathbf{C}(X \times \Omega)$ such that $f \succsim g$ iff

$$
\sum_{\omega \in \Omega} \mathbf{E}(U(\cdot, \omega), f(\omega)) \geq \sum_{\omega \in \Omega} \mathbf{E}(U(\cdot, \omega), g(\omega)) \quad \text { for every } U \in \mathcal{U}
$$

for any acts $f$ and $g$ in $\mathcal{F}$.

The "if" part of this assertion is straightforward, so we focus on its "only if" part alone. The argument for this part is a modification of the one given by Dubra, Maccheroni, and Ok (2004) for their expected multi-utility theorem. 
Let $\succsim$ be a preference relation on $\mathcal{F}$ that satisfies the Independence and Continuity Axioms. For any act $h$ in $\mathcal{F}$, define the Borel probability measure $\mathbf{h}$ on $X \times \Omega$ by

$$
\mathbf{h}(S \times\{\omega\}):=\frac{1}{|\Omega|} h(\omega)(S),
$$

for any $\omega \in \Omega$ and Borel subset $S$ of $X$. Let $\mathcal{S}:=\{\mathbf{h}: h \in \mathcal{F}\}$, which is a closed and convex subset of $\Delta(X \times \Omega)$. Next, we define the binary relation $\unrhd$ on $\mathcal{S}$ as

$$
\mathbf{f} \unrhd \mathbf{g} \quad \text { iff } \quad f \succsim g .
$$

It is easy to see that $\unrhd$ is affine. Furthermore, $\unrhd$ is a closed subset of $\Delta(X \times \Omega)^{2}$. These properties of $\unrhd$ allow us to borrow Lemma 2 of Dubra, Maccheroni, and Ok (2004):

Claim A.1: The set

$$
C(\unrhd):=\{\lambda(\mathbf{r}-\mathbf{s}): \lambda>0 \text { and } \mathbf{r} \unrhd \mathbf{s}\}
$$

is a convex cone in the linear space spanned by $\mathcal{S}$. Furthermore,

$$
\mathbf{f} \unrhd \mathbf{g} \quad \text { iff } \quad \mathbf{f}-\mathbf{g} \in C(\unrhd)
$$

for any $\mathbf{f}$ and $\mathbf{g}$ in $\mathcal{S}$.

Now let ca $(X \times \Omega)$ denote the normed linear space of signed finite Borel measures on $X \times \Omega$. While this space is normed by the total variation norm, it is isometrically isomorphic to $\mathbf{C}(X \times \Omega)^{*}$, because $X \times \Omega$ is compact. We use this duality to topologize $\mathrm{ca}(X \times \Omega)$ with the weak ${ }^{*}$-topology. For concreteness, let us denote the resulting topological space by ca $(X \times \Omega)_{*}$. (The notation $\mathrm{ca}(X)_{*}$ is similarly interpreted.) We note that, by definition, a net $\left(\mu_{\alpha}\right)$ in $\mathrm{ca}(X \times \Omega)_{*}$ converges to a signed finite Borel measure $\mu$ on $X \times \Omega$ iff

$$
\int_{X \times \Omega} U d \mu_{\alpha} \rightarrow \int_{X \times \Omega} U d \mu \quad \text { for all } U \in \mathbf{C}(X \times \Omega) .
$$

We shall show next that the cone $C(\unrhd)$ is closed in $\operatorname{ca}(X \times \Omega)_{*}$.

CLAIM A.2: $C(\unrhd)$ is a closed subset of $\operatorname{ca}(X \times \Omega)_{*}$.

PRoOF: The Krein-Smulian theorem says that every sequentially weak*closed convex set in the dual of a separable normed linear space is weak*closed. But, since $X \times \Omega$ is compact, $\mathbf{C}(X \times \Omega)$ is separable, and ca $(X \times \Omega)$ is isometrically isomorphic to $\mathbf{C}(X \times \Omega)^{*}$. It follows that it is enough to show that $C(\unrhd)$ is sequentially closed in $\operatorname{ca}(X \times \Omega)_{*}$ to establish Claim A.2. 
Take an arbitrary sequence $\left(\mu_{m}\right)$ in $C(\unrhd)$. By definition, there exist a sequence $\left(\lambda_{m}\right)$ of positive real numbers, and sequences $\left(\mathbf{f}^{m}\right)$ and $\left(\mathbf{g}^{m}\right)$ in $\mathcal{S}$ such that

$$
\mu_{m}=\lambda_{m}\left(\mathbf{f}^{m}-\mathbf{g}^{m}\right) \quad \text { and } \quad \mathbf{f}^{m} \unrhd \mathbf{g}^{m}
$$

for each positive integer $m$. Assume that $\left(\mu_{m}\right)$ converges in $\mathrm{ca}(X \times \Omega)_{*}$. Clearly, if $\mathbf{f}^{m}=\mathbf{g}^{m}$ for infinitely many $m$, then $\left(\mu_{m}\right)$ has a subsequence that converges to the origin of $\operatorname{ca}(X \times \Omega)$. As $C(\unrhd)$ contains the origin, we are done in this case. Let us assume then that $\mathbf{f}^{m}$ is distinct from $\mathbf{g}^{m}$ for all but finitely many $m$. Without loss of generality, we assume that $\mathbf{f}^{m} \neq \mathbf{g}^{m}$ for each $m$.

Note that, by definition,

$$
\left(\int_{X \times \Omega} U d \mu_{1}, \int_{X \times \Omega} U d \mu_{2}, \ldots\right)
$$

is a convergent real sequence for every $U$ in $\mathbf{C}(X \times \Omega)$. It follows that

$$
\sup \left\{\int_{X \times \Omega} U d \mu_{1}, \int_{X \times \Omega} U d \mu_{2}, \ldots\right\}<\infty \quad \text { for every } U \in \mathbf{C}(X \times \Omega) .
$$

By the uniform boundedness principle, therefore, $\sup \left\{\left\|\mu_{1}\right\|,\left\|\mu_{2}\right\|, \ldots\right\}<\infty$, that is, there exists a real number $K$ such that

$$
\left\|\lambda_{m}\left(\mathbf{f}^{m}-\mathbf{g}^{m}\right)\right\| \leq K
$$

for every $m$, where $\|\cdot\|$ is the total variation norm. On the other hand, by the Jordan decomposition theorem, for each positive integer $m$ and state $\omega$ in $\Omega$, there exist two mutually singular Borel probability measures $t^{m}(\omega)$ and $s^{m}(\omega)$ and a positive real number $\alpha_{m}(\omega)$ such that

$$
f^{m}(\omega)-g^{m}(\omega)=\alpha_{m}(\omega)\left(t^{m}(\omega)-s^{m}(\omega)\right) .
$$

Letting

$$
\alpha_{m}:=\max \left\{\alpha_{m}(\omega): \omega \in \Omega\right\}
$$

and

$$
r^{m}(\omega):=\frac{\alpha_{m}(\omega)}{\alpha_{m}} t^{m}(\omega)+\left(1-\frac{\alpha_{m}(\omega)}{\alpha_{m}}\right) s^{m}(\omega),
$$

we have

$$
f^{m}(\omega)-g^{m}(\omega)=\alpha_{m}\left(r^{m}(\omega)-s^{m}(\omega)\right)
$$


for each $m$ and $\omega$. Furthermore, for at least one state $\omega$ in $\Omega$, the probability measures $r^{m}(\omega)$ and $s^{m}(\omega)$ are mutually singular. Therefore, we have

$$
\mathbf{f}^{m}-\mathbf{g}^{m}=\alpha_{m}\left(\mathbf{r}^{m}-\mathbf{s}^{m}\right) \quad \text { and } \quad\left\|\mathbf{r}^{m}-\mathbf{s}^{m}\right\| \geq \frac{2}{|\Omega|},
$$

and hence,

$$
\begin{aligned}
\left\|\lambda_{m}\left(\mathbf{f}^{m}-\mathbf{g}^{m}\right)\right\| & =\left\|\lambda_{m} \alpha_{m}\left(\mathbf{r}^{m}-\mathbf{s}^{m}\right)\right\| \\
& =\lambda_{m} \alpha_{m}\left\|\mathbf{r}^{m}-\mathbf{s}^{m}\right\| \\
& \geq \frac{2 \lambda_{m} \alpha_{m}}{|\Omega|}
\end{aligned}
$$

for every $m$. Combining this observation with (7) shows that $\left(\lambda_{m} \alpha_{m}\right)$ is a bounded real sequence. Thus, there exists a strictly increasing sequence $\left(m_{k}\right)$ of positive integers such that $\left(\lambda_{m_{k}} \alpha_{m_{k}}\right)$ converges. Since $X$ is compact, Prokhorov's theorem entails that $\Delta(X)$ is a compact subset of ca $(X)$ relative to the topology of weak convergence, and hence, for every state $\omega$, both $r^{m_{k}}(\omega)$ and $s^{m_{k}}(\omega)$ must have convergent subsequences in ca $(X)_{*}$. Passing to these subsequences consecutively, we find a strictly increasing sequence $\left(n_{k}\right)$ of positive integers such that, for each state $\omega,\left(\lambda_{n_{k}} \alpha_{n_{k}}\right),\left(r^{n_{k}}(\omega)\right)$, and $\left(s^{n_{k}}(\omega)\right)$ converge, the latter two in ca $(X)_{*}$, to $\lambda, r(\omega)$, and $s(\omega)$, respectively. Obviously, $\lambda \geq 0$ and $\mathbf{r}$ and $\mathbf{s}$ are Borel probability measures on $X \times \Omega$. Moreover, for any $U \in \mathbf{C}(X \times \Omega)$, we have

$$
\begin{aligned}
\int_{X \times \Omega} U d \mathbf{r}^{n_{k}} & =\sum_{\omega \in \Omega} \int_{X \times\{\omega\}} U d \mathbf{r}^{n_{k}} \\
& =\sum_{\omega \in \Omega} \frac{1}{|\Omega|} \int_{X} U(\cdot, \omega) d r^{n_{k}}(\omega) \\
& \rightarrow \sum_{\omega \in \Omega} \frac{1}{|\Omega|} \int_{X} U(\cdot, \omega) d r(\omega) \\
& =\int_{X \times \Omega} U d \mathbf{r},
\end{aligned}
$$

and hence $\mathbf{r}^{n_{k}} \rightarrow \mathbf{r}$ in ca $(X \times \Omega)_{*}$, and similarly, $\mathbf{s}^{n_{k}} \rightarrow \mathbf{s}$ in ca $(X \times \Omega)_{*}$. Consequently,

$$
\mu_{m_{k}}=\lambda_{n_{k}}\left(\mathbf{f}^{n_{k}}-\mathbf{g}^{n_{k}}\right)=\lambda_{n_{k}} \alpha_{n_{k}}\left(\mathbf{r}^{n_{k}}-\mathbf{s}^{n_{k}}\right) \rightarrow \lambda(\mathbf{r}-\mathbf{s})
$$

as $k \rightarrow \infty$. But since $\mathbf{f}^{n_{k}} \unrhd \mathbf{g}^{n_{k}}$ and $\mathbf{r}^{n_{k}}-\mathbf{s}^{n_{k}}=\frac{1}{\alpha_{m}}\left(\mathbf{f}^{n_{k}}-\mathbf{g}^{n_{k}}\right)$, Claim A.1 implies that $\mathbf{r}^{n_{k}} \unrhd \mathbf{s}^{n_{k}}$ for each $k$. As $\unrhd$ is a closed subset of $\Delta(X \times \Omega)^{2}$, therefore, we 
have $\mathbf{r} \unrhd \mathbf{s}$. Thus $\lambda(\mathbf{r}-\mathbf{s}) \in C(\unrhd)$, that is, $\left(\mu_{n_{k}}\right)$ converges to a point in $C(\unrhd)$ relative to $\mathrm{ca}(X \times \Omega)_{*}$. Since every subsequence of a convergent sequence converges to the limit of the mother sequence, we are done.

Q.E.D.

Given that $C(\unrhd)$ is a closed convex cone in the locally convex topological linear space ca $(X \times \Omega)_{*}$, we may apply the separating hyperplane theorem to conclude that $C(\unrhd)$ equals the intersection of all closed halfspaces in ca $(X \times \Omega)_{*}$ that contains $C(\unrhd)$ and that goes through the origin. Consequently, there exist a set $\mathcal{L}$ of continuous linear functionals on ca $(X \times \Omega)_{*}$ and a real map $\sigma$ on $\mathcal{L}$ such that, for any $\mathbf{f}$ and $\mathbf{g}$ in $\mathcal{S}$, we have

$$
\mathbf{f} \unrhd \mathbf{g} \quad \text { iff } \quad L(\mathbf{f}-\mathbf{g}) \geq 0 \quad \text { for every } L \in \mathcal{L},
$$

that is,

$$
\mathbf{f} \unrhd \mathbf{g} \quad \text { iff } \quad L(\mathbf{f}) \geq L(\mathbf{g}) \quad \text { for every } L \in \mathcal{L} .
$$

Now, fix an $L$ in $\mathcal{L}$ arbitrarily, and define the binary relation $\succsim_{L}$ on $\mathcal{F}$ as

$$
f \succsim_{L} g \quad \text { iff } \quad L(\mathbf{f}) \geq L(\mathbf{g}) .
$$

It is easy to see that the linearity and continuity of $L$ ensure that $\succsim_{L}$ satisfies the Independence and Continuity Axioms. Since $\succsim_{L}$ is complete, therefore, we may apply the classical state-dependent expected utility theorem to conclude that there exists a map $U_{L} \in \mathbf{C}(X \times \Omega)$ such that, for every $f$ and $g$ in $\mathcal{F}$,

$$
f \succsim_{L} g \quad \text { iff } \quad \sum_{\omega \in \Omega} \int_{X} U_{L}(\cdot, \omega) d f(\omega) \geq \sum_{\omega \in \Omega} \int_{X} U_{L}(\cdot, \omega) d g(\omega) .
$$

Combining this representation with (8) and (9), invoking Claim A.1, and letting $\mathcal{U}$ be the convex hull of $\left\{U_{L}: L \in \mathcal{L}\right\}$, completes our proof.

\section{A.2. On the Uniqueness of the Single-Prior Expected Multi-Utility Representation}

We provide here the uniqueness counterpart of the representation obtained in Theorem 1. To state the associated result formally, we need a final bit of notation.

NOTATION: For any nonempty subset $\mathcal{U}$ of $\mathbf{C}(X)$, by $\langle\mathcal{U}\rangle$ we mean the closed and convex conical hull of $\mathcal{U}$ and the constant functions on $X$. That is, $v \in\langle\mathcal{U}\rangle$ iff either $v$ can be written as a positive linear combination of finitely many maps in $\mathcal{U}$ plus a constant, or it is equal to the (uniform) limit of a sequence of maps in $\mathbf{C}(X)$, each of which can be written this way.

The uniqueness of the notion of single-prior expected multi-utility representation is formulated as follows: 
THEOREM 3: Let $\succsim$ be a preference relation on $\mathcal{F}$ such that $\succsim \neq \mathcal{F} \times \mathcal{F}$. If $(\mu, \mathcal{U})$ and $(\nu, \mathcal{V})$ are two single-prior expected multi-utilities for $\succsim$, then $\mu=\nu$ and $\langle\mathcal{U}\rangle=\langle\mathcal{V}\rangle$.

The proof of this result is based on the following result, which is a consequence of the separating hyperplane theorem. A more general version of this result was proved by Dubra, Maccheroni, and Ok (2004), so we omit its proof here.

LEMMA: Let $X$ be a compact metric space. Two nonempty sets $\mathcal{U}$ and $\mathcal{V}$ in $\mathbf{C}(X)$ satisfy, for each $p$ and $q$ in $\Delta(X)$,

$$
\begin{aligned}
& \int_{X} u d p \geq \int_{X} u d q \text { for all } u \in \mathcal{U} \quad \text { iff } \\
& \int_{X} v d p \geq \int_{X} v d q \quad \text { for all } v \in \mathcal{V}
\end{aligned}
$$

if, and only if, $\langle\mathcal{U}\rangle=\langle\mathcal{V}\rangle$.

Now let $(\mu, \mathcal{U})$ and $(\nu, \mathcal{V})$ be two single-prior expected multi-utility representations for a preference relation $\succsim$, and assume that $\succsim \neq \mathcal{F} \times \mathcal{F}$. By the lemma above, we readily have $\langle\mathcal{U}\rangle=\langle\mathcal{V}\rangle$. Since $\succsim \neq \mathcal{F} \times \mathcal{F}$, we can find two lotteries $p$ and $q$ in $\Delta(X)$ and a map $v$ in $\mathcal{V}$ such that $\mathbf{E}(v, p)>\mathbf{E}(v, q)$. Now take an arbitrary $\omega$ in $\Omega$, and consider the acts

$$
f:=p \mathbf{1}_{\{\omega\}}+q \mathbf{1}_{\Omega \backslash\{\omega\}} \quad \text { and } \quad f^{\mu}:=(\mu(\omega) p+(1-\mu(\omega)) q) \mathbf{1}_{\Omega} .
$$

Then, for any $u$ in $\mathcal{U}$, we have

$$
\sum_{\tau \in \Omega} \mu(\tau) \mathbf{E}(u, f(\tau))=\sum_{\tau \in \Omega} \mu(\tau) \mathbf{E}\left(u, f^{\mu}(\tau)\right),
$$

whence $f \sim f^{\mu}$. But this implies that

$$
\sum_{\tau \in \Omega} \nu(\tau) \mathbf{E}(v, f(\tau))=\sum_{\tau \in \Omega} \nu(\tau) \mathbf{E}\left(v, f^{\mu}(\tau)\right)
$$

which can be true only if $\nu(\omega)=\mu(\omega)$. Since $\omega$ was chosen arbitrarily in $\Omega$, we may conclude that $\mu=\nu$, thereby completing the proof of Theorem 3 .

\section{REFERENCES}

Anscombe, F., AND R. Aumann (1963): "A Definition of Subjective Probability,” The Annals of Mathematical Statistics, 34, 199-205. [1791]

AumanN, R. (1962): "Utility Theory Without the Completeness Axiom," Econometrica, 30, 445-462. [1791,1794] 
Bewley, T. (2002): "Knightian Decision Theory. Part I," Decisions in Economics and Finance, 25, 79-110. [1791,1794]

Dubra, J., F. MACCHERONI, AND E. A. OK (2004): "Expected Utility Theory Without the Completeness Axiom," Journal of Economic Theory, 115, 118-133. [1795,1802,1803,1807]

GAlAABAATAR, T., AND E. KARNI (2012): "Subjective Expected Utility With Incomplete Preferences," Econometrica (forthcoming). [1799,1800]

GARCía DEL AMO, A., AND D. Ríos InSUA (2002): "A Note on an Open Problem in the Foundation of Statistics," Revista de la Real Academia de Ciencias Exactas, Fisicas y Naturales. Serie A. Mathemáticas, 96, 55-61. [1792,1800]

GHIRARDATO, P., AND J. KATZ (2006): "Indecision Theory: Quality of Information and Voting Behavior," Journal of Public Economics, 8, 379-399. [1794]

KeLSEY, D., AND E. YALCIN (2007): "The Arbitrage Pricing Theorem With Incomplete Preferences," Mathematical Social Sciences, 54, 90-105. [1794]

KREPS, D. (1988): Notes on the Theory of Choice. Boulder: Westview Press. [1800]

Lopomo, G., L. RigotTi, AND C. Shannon (2009): "Uncertainty in Mechanism Design,” Report, Duke University. [1794]

MACHINA, M., AND D. SCHMEIDLER (1992): "A More Robust Definition of Subjective Probability," Econometrica, 60, 745-780. [1795]

NAU, R. (2006): “The Shape of Incomplete Preferences,” The Annals of Statistics, 34, 2430-2448. $[1792,1799,1800]$

RIGOTTI, L., AND C. SHANNON (2005): "Uncertainty and Risk in Financial Markets," Econometrica, 73, 203-243. [1794]

SEIDENFELD, T., M. SCHERVISH, AND J. KADANE (1995): "A Representation of Partially Ordered Preferences," The Annals of Statistics, 23, 2168-2217. [1792,1800]

Dept. of Economics, New York University, 269 Mercer Street, New York, NY 10012,U.S.A.; efe.ok@nyu.edu,

Division of the Humanities and Social Sciences, California Institute of Technology, 1200 E California Blvd, Pasadena, CA 91105, U.S.A.; ortoleva@caltech.edu, and

Departamento de Economia, Universidade de Brasília, Campus Darcy Ribeiro, Brasília, DF 70910-900, Brazil; riella@unb.br.

Manuscript received July, 2008; final revision received October, 2011. 